Check for updates

Cite this: RSC Adv., 2022, 12, 3191

\title{
Synthesis and characterization of naphthalene derivatives for two-component heterojunction- based ambipolar field-effect transistors complemented with copper hexadecafluorophthalocyanine ( $\left.F_{16} \mathrm{CuPc}\right)$
}

\begin{abstract}
Guangjin Chen, $\dagger^{\mathrm{a}}$ Xinwei Huo, (D) $\dagger^{\mathrm{a}}$ Qingfang Ma, ${ }^{\text {a }}$ Qinghua Pan, ${ }^{\mathrm{a}}$ Hanghong Fan, ${ }^{a}$ Wangjing $\mathrm{Ma},{ }^{* b}$ Renren Fang, ${ }^{a}$ Ru Chen ${ }^{\mathrm{a}}$ and Jianhua Gao (D) *a

In order to develop organic semiconductor materials with good performance, herein, a series of naphthalene derivatives were designed and synthesized by a "building-blocks approach" connected through $\alpha$-bond, double bond, and triple bond, respectively. Thin-film transistors were fabricated in single-component and two-component modes based on these naphthalene derivatives by combining the $\mathrm{F}_{16} \mathrm{CuPc}$ as the $\mathrm{n}$-type material. The ambipolar performance was investigated by adjusting the device preparation procedure with the hole and electron mobility of up to $10^{-2} \mathrm{~cm}^{2} \mathrm{~V}^{-1} \mathrm{~s}^{-1}$. Furthermore, the electrical performance was also improved to $0.73 \mathrm{~cm}^{2} \mathrm{~V}^{-1} \mathrm{~s}^{-1}$ using the two-component bilayer configuration.
\end{abstract}

Received 1st November 2021

Accepted 28th December 2021

DOI: 10.1039/d1ra08022a

rsc.li/rsc-advances and coworkers reported the first ambipolar OFET device by means of a bilayer technique based on p-type $\alpha$-hexathienylene and n-type $\mathrm{C}_{60}$ semiconductors, ${ }^{12}$ the two-component bilayer mode has become an important alternative and attracted significant research interest, although it is even more difficult to understand its working mechanism than the single-component mode. A series of two-component bilayer OFETs were investigated by combining p-type and n-type organic semiconductors, including small molecules and even polymers such as phenacene/perylene, ${ }^{13}$ pentacene/PTCDI- $\mathrm{C}_{13} \mathrm{H}_{27},{ }^{14}$ pentacene $/ \mathrm{C}_{60},{ }^{15}$ $\mathrm{BP}_{2} \mathrm{~T} / \mathrm{F}_{16} \mathrm{CuPc}^{16}$ and $\mathrm{CuPc} / \mathrm{F}_{16} \mathrm{CuPc},{ }^{17}$ etc. In particular, fluorinated metal phthalocyanines, such as hexadecafluorophthalocyanine $\left(\mathrm{F}_{16} \mathrm{CuPc}\right)$, have demonstrated potential advantages in constructing ambipolar OFETs as typical n-type organic semiconductors due to their excellent electrical and optical properties as well as air-stable properties. ${ }^{\mathbf{1 6 - 1 9}}$

Naphthalene is widely used in the synthesis of organic photo-electronic materials as a building block due to its stable structure, cheap price and easy halogenations at the 2- and 6positions. ${ }^{20-26}$ Its bromides, such as 2-bromonaphthalene and 2,6-dibromonaphthalene, are easily and commercially available, which is conducive to future industrialization. The "building-blocks approach" is one important way to develop organic semiconductor materials. ${ }^{27}$ By the combination of various building blocks, new molecules can be produced conveniently. Moreover, the mode of connection, which includes $\alpha$-, double and triple bonds, etc., could significantly affect the material properties. ${ }^{28-33}$
${ }^{a}$ College of Material, Chemistry and Chemical Engineering, Key Laboratory of Organosilicon Chemistry and Material Technology, Ministry of Education, Hangzhou Normal University, Hangzhou, 311121, Zhejiang, People's Republic of China. E-mail: gaojh@hznu.edu.cn; maqf@hznu.edu.cn; Fax: +86-571-28865540; Tel: +86571-28865540

${ }^{b}$ Technical Institute of Physics and Chemistry, Chinese Academy of Sciences, Beijing 100190, P. R. China.E-mail: wjma@mail.ipc.ac.cn

$\dagger$ Guangjin Chen and Xinwei Huo contributed equally to this work. 
In order to develop organic semiconductor materials with good performance, herein, a series of naphthalene derivatives were designed and synthesized by the "building-blocks approach," connected through the $\alpha$-bond, double bond and triple bond, respectively. Thin-film transistor devices were fabricated based on these compounds, and the highest mobility of up to $0.53 \mathrm{~cm}^{2} \mathrm{~V}^{-1} \mathrm{~s}^{-1}$ was achieved for the single-component devices. Furthermore, two-component bilayer OFETs devices were prepared by combining the naphthalene derivatives with $\mathrm{F}_{16} \mathrm{CuPc}$ as the n-type material. The ambipolar performance was investigated by adjusting the bilayer configuration, and the electrical performance was also improved by the device preparation procedure.

\section{Results and discussion}

\section{Synthesis}

The synthetic routes of naphthalene derivatives 2,6-di $\left(1 H^{-}\right.$ inden-2-yl) naphtha-ene (DIN), 2,6-di((E)-styry)naphthalene (DSN) and 2,6-bis(phenylthyny)naphtha-ene (DPEN) are described in Scheme 1. Compounds DSN and DIN were synthesized directly through Suzuki coupling reaction between 2,6-dibromonaphthalene and boric acid intermediate. Compound DPEN was synthesized through a Sonogashira coupling reaction between 2,6-dibromonaphthalene and ethynylbenzene. Purification was carried out by column chromatography, followed by recrystallization, and the chemical structure was thoroughly characterized by mass spectrometry, ${ }^{1} \mathrm{H}-\mathrm{NMR}$ and elemental analysis.

\section{Thermal, optical and electrochemical properties}

The thermal properties of the naphthalene derivatives were measured by thermogravimetric analysis (TGA) under nitrogen atmosphere, as shown in Fig. 1 and Table 1. When the weight loss was about $5 \%$, the thermal decomposition temperatures of DIN, DSN and DPEN were $350{ }^{\circ} \mathrm{C}, 300{ }^{\circ} \mathrm{C}$ and $250{ }^{\circ} \mathrm{C}$, respectively, indicating that all the derivatives showed good thermal stability, and that the introduction of unsaturated bonds reduced the thermal stability of the compounds.

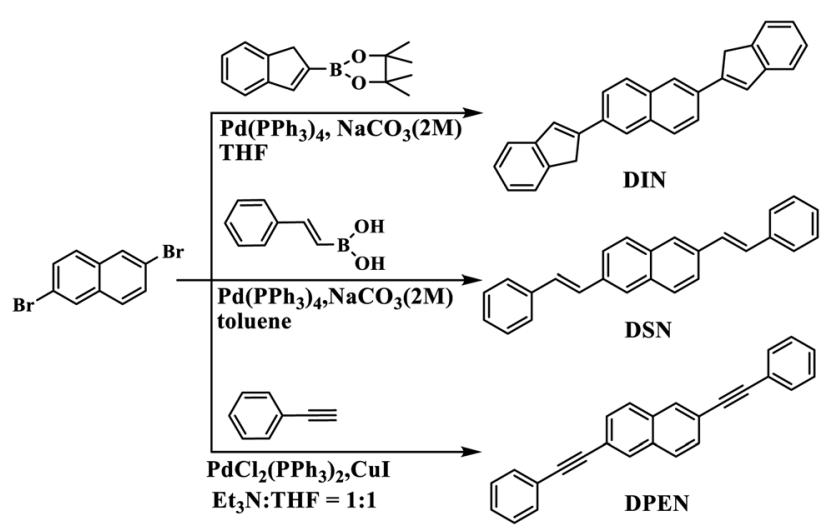

Scheme 1 Synthetic routes for naphthalene derivatives.

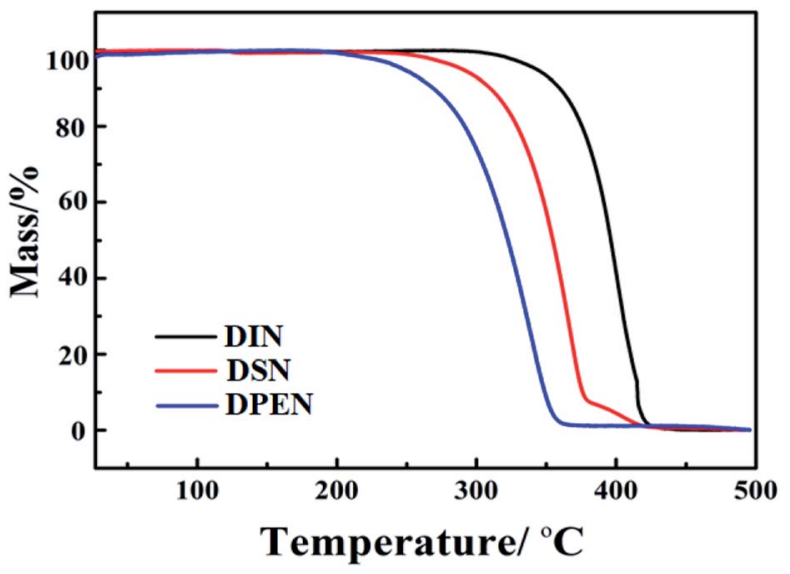

Fig. 1 TGA results of the naphthalene derivatives.

The optical properties of the naphthalene derivatives were investigated by UV-vis absorption spectroscopy. As shown in Fig. 2 and Table 1, the maximum absorption peak of the compounds DIN, DSN and DPEN in $\mathrm{CH}_{2} \mathrm{Cl}_{2}$ solution are located at $339 \mathrm{~nm}, 359 \mathrm{~nm}$ and $354 \mathrm{~nm}$, respectively, which correspond to the $\pi-\pi$ transition band of the three compounds. According to the initial absorption wavelengths, the band gaps are $3.16 \mathrm{eV}$, $3.09 \mathrm{eV}$ and $3.38 \mathrm{eV}$, respectively, which were calculated using the equation $E_{\mathrm{g}}=1240 / \lambda_{\text {onset }}$. Compared with that in solution, the spectral absorption of DIN vacuum-deposited thin film on a quartz substrate was red-shifted, and the thin films of DPEN and DSN were blue-shifted.

The redox properties of the naphthalene derivatives were measured in $\mathrm{CH}_{2} \mathrm{Cl}_{2}$ solution by cyclic voltammetry (CV). As shown in Fig. 3 and Table 1, the HOMO levels of DIN, DSN and DPEN are $-5.53 \mathrm{eV},-5.66 \mathrm{eV}$ and $-5.98 \mathrm{eV}$, respectively, which were calculated by the formula $E_{\mathrm{HOMO}}=-4.8+E_{\mathrm{Fc}}-E_{\mathrm{Ox}}$ using ferrocene as internal standard. ${ }^{34,35}$ Then, by combining with their optical band gaps, the LUMO energy levels were calculated to be $-2.37 \mathrm{eV},-2.57 \mathrm{eV}$ and $-2.60 \mathrm{eV}$, respectively. Furthermore, the molecular-orbital calculations of the HOMO and LUMO levels were performed using the density functional

Table 1 Thermal, photophysical, and electrochemical properties and the calculated energy levels of the naphthalene derivatives ${ }^{a}$

\begin{tabular}{llll}
\hline Compds & DIN & DSN & DPEN \\
\hline$T_{\mathrm{d}} /{ }^{\circ} \mathrm{C}$ & 350 & 300 & 250 \\
$\lambda_{\text {onset,ox }} / \mathrm{nm}$ & 393 & 401 & 367 \\
$E_{\mathrm{g}, \mathrm{op}} / \mathrm{eV}$ & 3.16 & 3.09 & 3.38 \\
$E_{\text {Ox, } 1 / 2}$ & 1.13 & 1.26 & 1.58 \\
$\mathrm{HOMO}^{\mathrm{a}} / \mathrm{eV}$ & -5.53 & -5.66 & -5.98 \\
$\mathrm{LUMO}^{\mathrm{a}} / \mathrm{eV}$ & -2.37 & -2.57 & -2.60 \\
$\mathrm{HOMO}^{\mathrm{b}} / \mathrm{eV}$ & -5.02 & -5.12 & -5.36 \\
$\mathrm{LUMO}^{\mathrm{b}} / \mathrm{eV}$ & -1.76 & -1.88 & -1.82 \\
$E_{\mathrm{g}} \mathrm{b} / \mathrm{eV}$ & 3.26 & 3.24 & 3.54
\end{tabular}

${ }^{a} E_{\mathrm{g}, \mathrm{op}}{ }^{\mathrm{a}}$ was determined from the onset of the UV-vis absorption spectra in solution. $\mathrm{HOMO}^{\mathrm{a}}=-4.8+E_{\mathrm{Fc}}-E_{\mathrm{ox}, 1 / 2}$; $\mathrm{LUMO}^{\mathrm{a}}=\mathrm{HOMO}+E_{\mathrm{g}, \mathrm{op}}$. $\mathrm{HOMO}^{\mathrm{b}}$ and $\mathrm{LUMO}^{\mathrm{b}}$ are based on the DFT (B3LYP/6-31G(d)) calculations. 


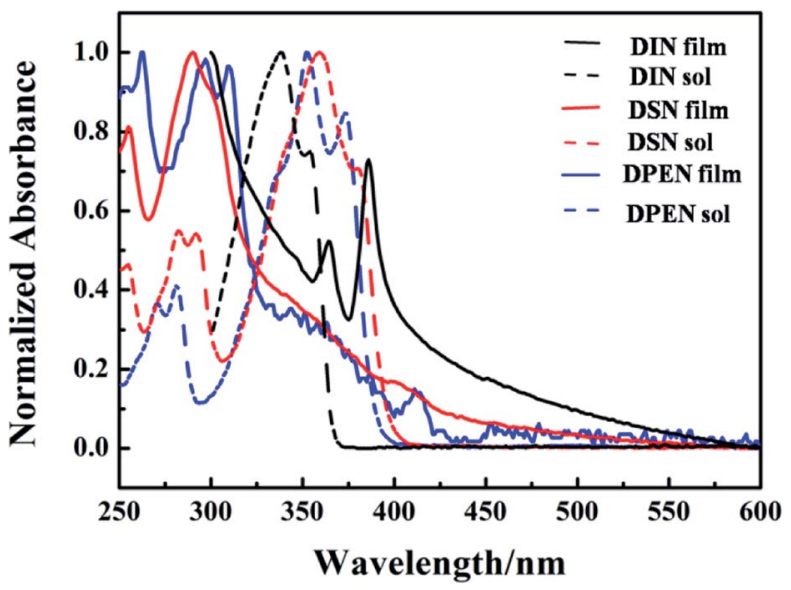

Fig. 2 Normalized UV-vis absorption spectra of the naphthalene derivatives in $\mathrm{CH}_{2} \mathrm{Cl}_{2}$ solution and as thin films.
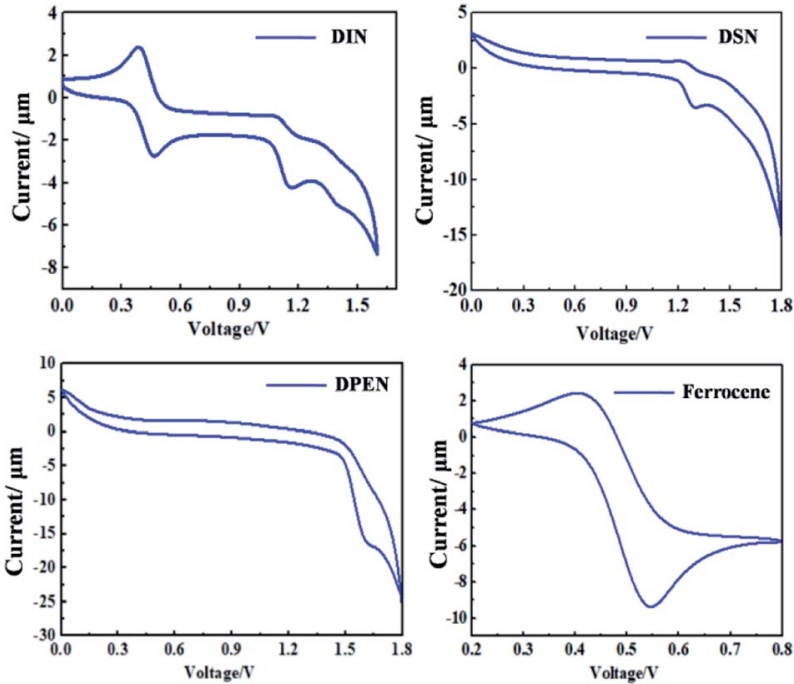

Fig. 3 Cyclic voltammograms of the naphthalene derivatives.
LUMO

DIN

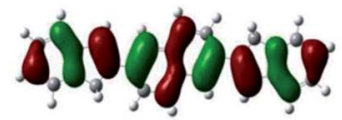

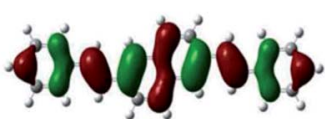

DPEN

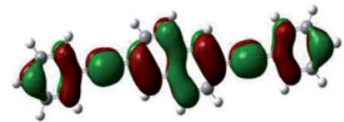

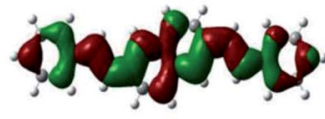
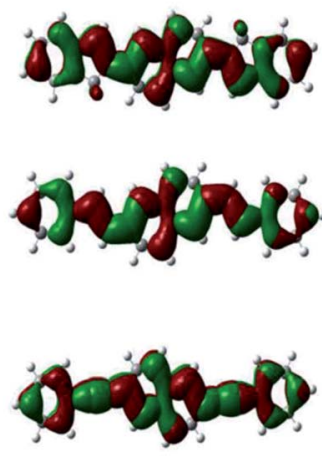

Fig. $4 \mathrm{HOMO}$ and LUMO orbitals of the naphthalene derivatives obtained using DFT calculations.

theory (DFT) method (B3LYP,6-13G(d)) to confirm the redox properties of the naphthalene derivatives (see Fig. 4). The simulated results also imply the good oxidation stability of DIN, DSN and DPEN.

\section{Thin film crystallinity and morphology}

The morphology and crystallinity of thin films are closely related to the charge transfer performance. Thin films of the naphthalene derivatives were deposited onto a octadecyltrichlorosilane (OTS) modified silicon wafer by vacuum evaporation and were characterized using atomic force microscopy (AFM) and X-ray diffraction (XRD). As shown in Fig. 5, at room temperature $\left(T_{\text {sub }}=20^{\circ} \mathrm{C}\right)$, the crystal grain of DIN is granulous and DSN is clustered; both grain sizes are small. DPEN forms a sheet-like shape, and the size even reaches $2 \mu \mathrm{m}$, but the grain boundary is also obviously large. With an increase in the substrate temperature to $60{ }^{\circ} \mathrm{C}$, all the grain sizes become larger, and the grain boundary becomes smaller, indicating that the semiconductor layers present better consecutiveness and less traps, which are conducive to the transport of carriers; thus the electrical performance of the device could be improved. When the substrate temperature was raised to $90{ }^{\circ} \mathrm{C}$, the grain size of DIN was significantly reduced, DSN was destroyed, and the grain boundary of DPEN was enlarged, which is usually unfavorable for OFET performance.

XRD measurements of the thin films deposited at different temperatures showed similar primary diffraction peaks at $2 \theta=$ $4.46^{\circ}, 4.35^{\circ}$ and $4.34^{\circ}$ for DIN, DSN and DPEN, respectively (see Fig. 6). The primary peaks showed distinct diffraction, and the corresponding $d$-spacings were $1.949 \mathrm{~nm}, 2.028 \mathrm{~nm}$ and $2.037 \mathrm{~nm}$, respectively. The values of the $d$-spacings are very close to the length of the molecules, which indicates that the molecules are oriented almost perpendicularly to the
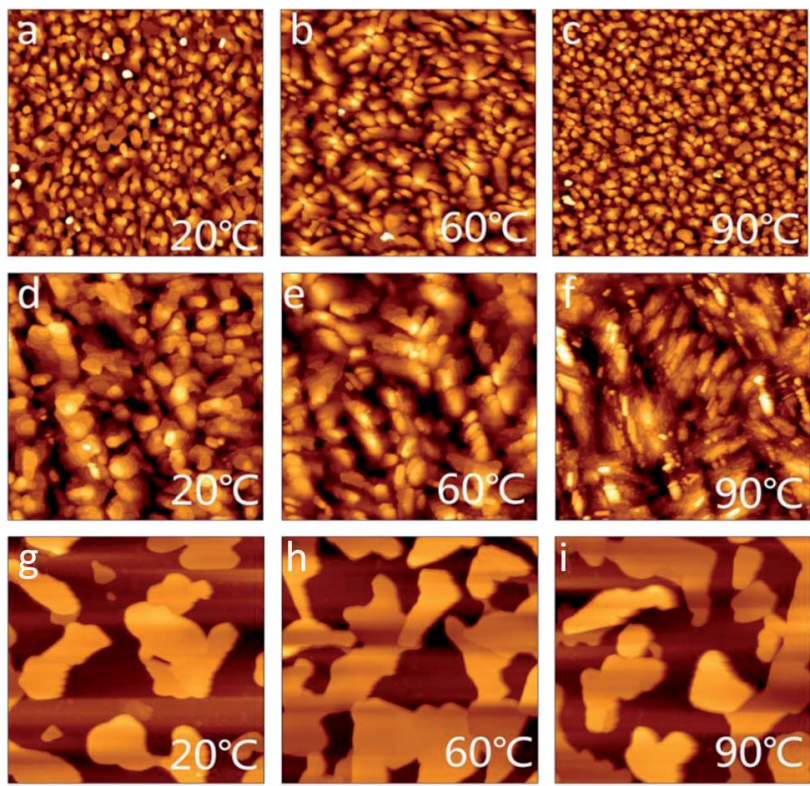

Fig. 5 AFM images $(5 \mu \mathrm{m} \times 5 \mu \mathrm{m})$ of the thin films of DIN $(\mathrm{a}-\mathrm{c})$, DSN $(d-f)$ and DPEN $(g-i)$ deposited at different temperatures. 

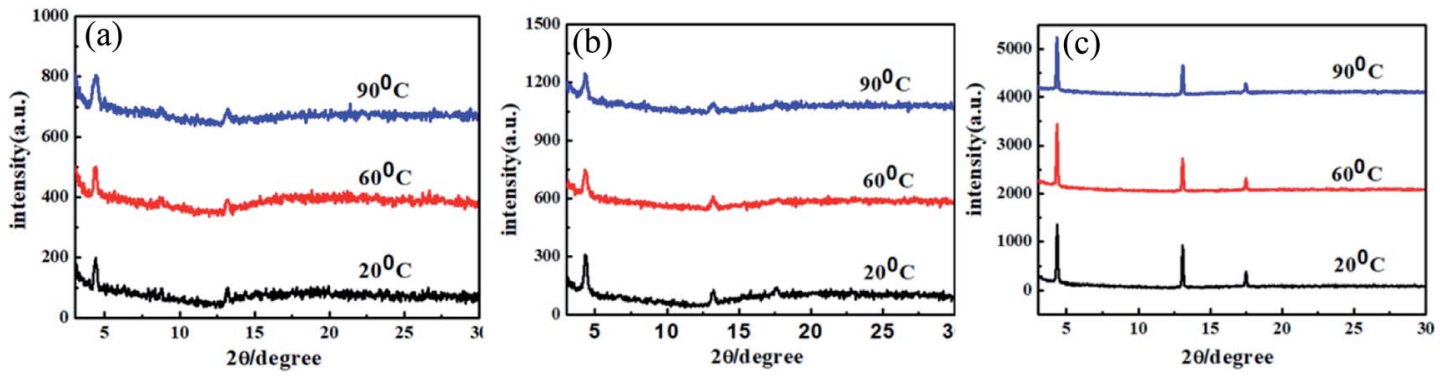

Fig. 6 X-ray diffraction of the thin films of (a) DIN, (b) DSN and (c) DPEN deposited at different temperatures.

substrate and that the $\pi-\pi$ stacking direction is parallel to the substrate.

\section{OFET performance}

Thin-film transistors of DIN, DSN and DPEN were fabricated in top-contact configuration using gold as source and drain electrodes, and doped silicon as the gate electrode. All the devices showed typical p-channel FET properties under ambient conditions. DSN exhibits excellent OFET performance, which is higher by one to two orders of magnitude than that of the other two materials. With increasing substrate temperature, the performance of DIN, DSN and DPEN improved. When the substrate temperature was increased to $60{ }^{\circ} \mathrm{C}$, the device performance was highest, and the mobility of DIN, DSN and DPEN reached up to $5.40 \times 10^{-2} \mathrm{~cm}^{2} \mathrm{~V}^{-1} \mathrm{~s}^{-1}, 0.53 \mathrm{~cm}^{2} \mathrm{~V}^{-1} \mathrm{~s}^{-1}$ and $3.42 \times 10^{-2} \mathrm{~cm}^{2} \mathrm{~V}^{-1} \mathrm{~s}^{-1}$, respectively. Fig. 7 shows the transfer and output curves of DIN, DSN and DPEN at $T_{\text {sub }}=$ $60{ }^{\circ} \mathrm{C}$, and the OFET performance obtained at different substrate temperatures is summarized in Table 2 . With further increasing the substrate temperature $\left(T_{\text {sub }}=90{ }^{\circ} \mathrm{C}\right)$, the
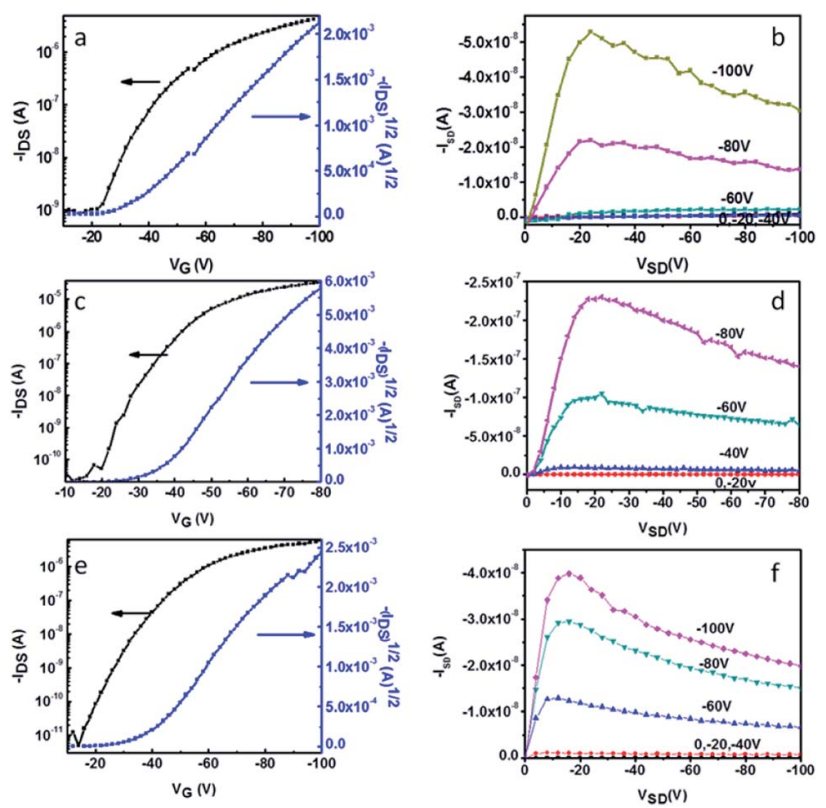

Fig. 7 Typical transfer (left) and out (right) curve of DIN (a and b), DSN (c and d) and DPEN (e and f). mobility decreased dramatically, which could be caused by the worse morphologies of the films evidenced by AFM.

To obtain ambipolar OFET performance, DSN was used as the p-type active semiconductor material and $\mathrm{F}_{16} \mathrm{CuPc}$ as the $\mathrm{n}$ type semiconductor to construct bilayer heterojunction devices. Both materials were put into a vacuum chamber and deposited onto OTS-modified silicon wafer successively. The substrate temperature was kept at $60{ }^{\circ} \mathrm{C}$ and without oxygen contact during the evaporation switching. The preparation procedure of the gold electrode was the same as the above unipolar devices. The $\mathrm{F}_{16} \mathrm{CuPc}$ was deposited firstly onto the silicon wafer at different thicknesses, and DSN was then deposited up to $15 \mathrm{~nm}$.

When the thickness of $\mathrm{F}_{16} \mathrm{CuPc}$ is $2 \mathrm{~nm}$ or $6 \mathrm{~nm}$, the transistors exhibit obvious ambipolar properties, with hole and electronic mobilities of up to $10^{-2} \mathrm{~cm}^{2} \mathrm{~V}^{-1} \mathrm{~s}^{-1}$ (see Fig. 8 and Table 3). With the increasing thickness of $\mathrm{F}_{16} \mathrm{CuPc}$, the electronic mobility increased, but the hole transport characteristics disappeared. For contrast, the DSN was deposited firstly, and $\mathrm{F}_{16} \mathrm{CuPc}$ was then deposited up to $10 \mathrm{~nm}$. When the thickness of DSN is $2 \mathrm{~nm}$ or $6 \mathrm{~nm}$, the transistors only exhibit p-type properties. With the thickness of DSN increased to $10 \mathrm{~nm}$, the transistors exhibit obvious ambipolar properties, with hole and electronic mobilities of up to $0.46 \mathrm{~cm}^{2} \mathrm{~V}^{-1} \mathrm{~s}^{-1}$ and $2.5 \times 10^{-3}$ $\mathrm{cm}^{2} \mathrm{~V}^{-1} \mathrm{~s}^{-1}$, respectively. It is worth noting that the hole mobility increased to $0.73 \mathrm{~cm}^{2} \mathrm{~V}^{-1} \mathrm{~s}^{-1}$ when the thickness of $\mathrm{F}_{16} \mathrm{CuPc}$ was reduced to $5 \mathrm{~nm}$, and the electronic mobility lowered by one order of magnitude. In this case, $\mathrm{F}_{16} \mathrm{CuPc}$ could mainly act as a dopant, not only as n-type acceptor material. Furthermore, DSN and $\mathrm{F}_{16} \mathrm{CuPc}$ were blended and coevaporated onto an OTS-modified silicon wafer, and the

Table 2 OFET characteristics of the naphthalene derivatives deposited at different annealing temperatures

\begin{tabular}{lllll}
\hline Compds & $T_{\text {sub }}{ }^{\circ} \mathrm{C}$ & $\mu\left(\mathrm{cm}^{2} \mathrm{~V}^{-1} \mathrm{~s}^{-1}\right)$ & $V_{\mathrm{t}}[\mathrm{V}]$ & On/off ratio \\
\hline DIN & $\mathrm{rt}$ & $4.80 \times 10^{-3}$ & -42 & $6 \times 10^{3}$ \\
& 60 & $5.40 \times 10^{-2}$ & -38 & $1 \times 10^{6}$ \\
& 90 & $6.25 \times 10^{-3}$ & -45 & $1 \times 10^{4}$ \\
DSN & $\mathrm{rt}$ & 0.17 & -29 & $4 \times 10^{4}$ \\
& 60 & 0.53 & -35 & $8 \times 10^{6}$ \\
& 90 & 0.21 & -47 & $1 \times 10^{4}$ \\
DPEN & $\mathrm{rt}$ & $6.80 \times 10^{-3}$ & -42 & $3 \times 10^{3}$ \\
& 60 & $3.42 \times 10^{-2}$ & -36 & $1 \times 10^{4}$ \\
& 90 & $8.1 \times 10^{-4}$ & -38 & $1 \times 10^{3}$
\end{tabular}



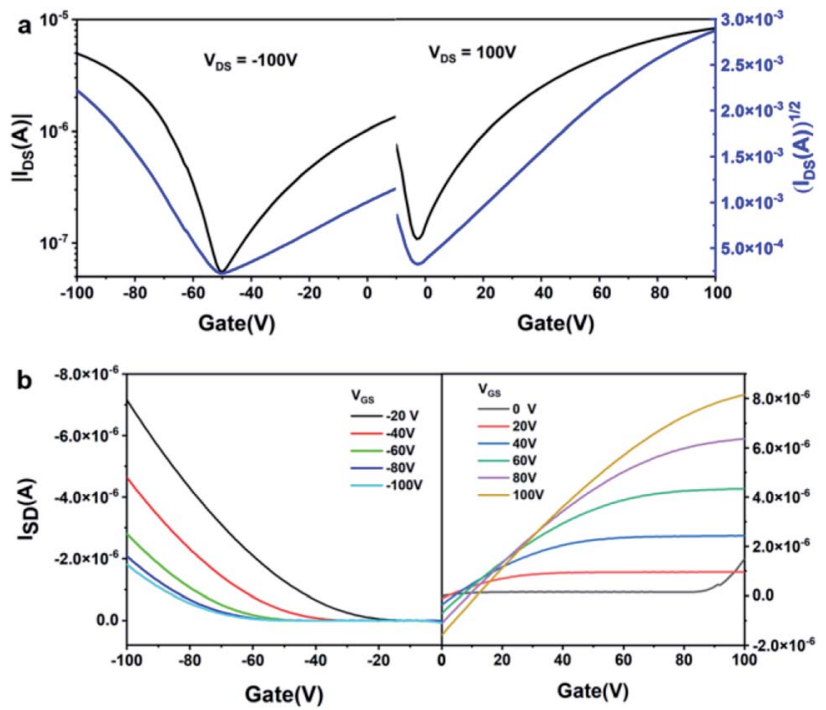

Fig. 8 Typical transfer (a) and out (b) curves of the ambipolar OFETs based on the DSN/F ${ }_{16}$ CuPc bilayer heterojunction devices.

Table 3 OFET characteristics of two-component bilayer heterojunction-based transistors with different configurations and thicknesses of $D S N / F_{16}$ CuPc thin films

\begin{tabular}{llllll}
\hline $\mathrm{DSN} / \mathrm{F}_{16} \mathrm{CuPc}$ & $\mu_{\mathrm{e}}$ & $\mu_{\mathrm{h}}$ & $\mathrm{F}_{16} \mathrm{CuPc} / \mathrm{DSN}$ & $\mu_{\mathrm{e}}$ & $\mu_{\mathrm{h}}$ \\
\hline $15 / 2$ & $7.5 \times 10^{-3}$ & 0.01 & $10 / 2$ & No & 0.042 \\
$15 / 6$ & 0.023 & 0.065 & $10 / 6$ & No & 0.063 \\
$15 / 10$ & 0.031 & No & $10 / 10$ & $2.5 \times 10^{-3}$ & 0.46 \\
$15 / 15$ & 0.048 & No & $5 / 10$ & $2.2 \times 10^{-4}$ & 0.73
\end{tabular}

The unit of thickness of the semiconductor layer is $\mathrm{nm}$. The unit of mobility is $\mathrm{cm}^{2} \mathrm{~V}^{-1} \mathrm{~s}^{-1}$.

obtained transistors only display p-channel FET properties with mobility up to $7.4 \times 10^{-2} \mathrm{~cm}^{2} \mathrm{~V}^{-1} \mathrm{~s}^{-1}$, and the electronic transport characteristics could not be detected. These results demonstrate that the ambipolar electrical performance could be effectively regulated by the device configuration and film thickness.

\section{Experimental}

\section{General}

All reagents and chemicals were purchased from J\&K Scientific and Aladdin and were used without further purification. ${ }^{1} \mathrm{H}$ NMR spectra were recorded on a Bruker DRX-400 Spectrometer with $\mathrm{Si}\left(\mathrm{CH}_{3}\right)_{4}$ as an external standard. Mass spectrometry (MS) was performed on a MicroTOF-Q II. Elemental analyses were performed by the CRMPO. The UV-vis spectrum was obtained on a TU-1901 double-beam UV-vis spectrophotometer. Thermogravimetric analysis (TGA) was carried out on a TAQ500. Cyclic voltammetry (CV) was performed on a CHI660C electrochemistry station in a dichloromethane solution using $0.1 \mathrm{M}$ tetrabutylammonium hexafluorophosphate $\left(\mathrm{Bu}_{4} \mathrm{NPF}_{6}\right)$ as supporting electrolyte at a scan rate of $100 \mathrm{mV} \mathrm{s}^{-1}$, using glass carbon as working electrode, Pt wire as counter electrode and $\mathrm{Ag} / \mathrm{AgCl}$ as reference electrode. AFM images of the film morphology were obtained using an atomic force microscope in tapping mode. XRD measurements were carried out in reflection mode using a PAN analytical X'pert powder diffraction system. The electrical characteristics of the devices were examined using a Keithley 4200 semiconductor parameter analyzer at room temperature in air.

\section{Thin-film transistor device fabrication}

OFET devices were fabricated by the bottom-gate/top-contact geometry configuration. A $50 \mathrm{~nm}$ thick organic semiconductor film was deposited onto the OTS-modified $\mathrm{SiO}_{2}$ substrate by vacuum evaporation at different substrate temperatures. Gold electrodes were deposited using a copper shadow mask with the channel length and width of 31 and $273 \mu \mathrm{m}$, respectively. The field effect properties were measured under ambient conditions. For the two-component bilayer devices, the thickness of semiconductor materials is listed in Table 3, and the thickness of the gold electrode is $20 \mathrm{~nm}$.

\section{Synthesis}

2,6-di(1H-inden-2-yl)naphthalene (DIN). 2,6-Dibromonaphthalene (572 $\mathrm{mg}, 2 \mathrm{mmol}$ ) was added to a $250 \mathrm{ml}$ round-bottom flask. After it was degassed and purged with nitrogen, $80 \mathrm{ml}$ of dry tetrahydrofuran solution was added, then $1 \mathrm{H}$-indole-2boronic acid pinacol ester (1000 mg, $4.13 \mathrm{mmol}$ ) and $\mathrm{Pd}\left(\mathrm{PPh}_{3}\right)_{4}(231 \mathrm{mg}, 0.2 \mathrm{mmol})$ were quickly added. After again purging with nitrogen, a solution of $\mathrm{Na}_{2} \mathrm{CO}_{3}(2 \mathrm{M}, 40 \mathrm{ml})$ was added, and the mixture was heated to reflux for $48 \mathrm{~h}$. After the reaction was completed, the mixture was cooled to room temperature and filtered, and the precipitate was collected and washed with water and methanol, sequentially. After purification by silica gel column chromatography, the title product was recrystallized from chloroform to give $330 \mathrm{mg}$ of pure compound DIN (yield 46\%). MS (MALDI-TOF): 356.0. ${ }^{1} \mathrm{H}$ NMR $\left(400 \mathrm{MHz}, \mathrm{CDCl}_{3}\right): \delta 7.97(\mathrm{~m}, 2 \mathrm{H}), 7.84(\mathrm{~m}, 4 \mathrm{H}), 7.52(\mathrm{~m}, 4 \mathrm{H})$, $7.37(\mathrm{~m}, 4 \mathrm{H}), 7.00(\mathrm{~s}, 2 \mathrm{H}), 3.94(\mathrm{~s}, 4 \mathrm{H})$. Anal. calcd for $\mathrm{C}_{28} \mathrm{H}_{20}: \mathrm{C}$, 94.34; H, 5.66. Found: C, 92.92; H, 5.53.

2,6-di((E)-styryl)naphthalene (DSN). 2,6-dibromonaphthalene (572 mg, $2 \mathrm{mmol}$ ) was added to a $250 \mathrm{ml}$ round-bottom flask and evacuated by vacuum, then $60 \mathrm{ml}$ of dry toluene solution was added. Under nitrogen condition, trans-BETAstyrene boronic acid (600 $\mathrm{mg}, 4 \mathrm{mmol})$ and $\mathrm{Pd}\left(\mathrm{PPh}_{3}\right)_{4}$ (231 $\mathrm{mg}, 0.2 \mathrm{mmol}$ ) were quickly added and purged with nitrogen. After $\mathrm{Na}_{2} \mathrm{CO}_{3}$ aqueous solution (2 M, $25 \mathrm{ml}$ ) was added, the mixture was heated and refluxed for $48 \mathrm{~h}$. After completion of the reaction, the mixture was cooled to room temperature. The crude product was purified by silica gel column chromatography and recrystallized from chloroform to give $276 \mathrm{mg}$ of pure compound DSN (yield 42\%). MS (MALDITOF): 332.0. ${ }^{1} \mathrm{H}$ NMR (400 MHz, $\left.\mathrm{CDCl}_{3}\right): \delta 7.82(\mathrm{~s}, 2 \mathrm{H}), 7.74$ (d, 2H), 7.57 (d, 2H), $7.52(\mathrm{~m}, 4 \mathrm{H}), 7.39(\mathrm{~m}, 4 \mathrm{H}), 7.30(\mathrm{~m}, 2 \mathrm{H})$, $7.08(\mathrm{~d}, 4 \mathrm{H})$. Anal. Calcd for $\mathrm{C}_{26} \mathrm{H}_{20}$ : C, 93.94; H, 6.06. Found: $\mathrm{C}$, 89.96; H, 5.88. 
2,6-Di(phenylethynyl)naphthalene (DPEN). 2,6-Dibromonaphthalene (572 mg, $2 \mathrm{mmol}), \mathrm{PdCl}_{2}\left(\mathrm{PPh}_{3}\right)_{2}$ (90 mg, 0.128 $\mathrm{mmol}$ ) and $\mathrm{CuI}(24 \mathrm{mg}, 0.126 \mathrm{mmol}$ ) were added to a round flask. A solution of $20 \mathrm{ml}$ of triethylamine and $20 \mathrm{ml}$ of anhydrous tetrahydrofuran was added. Then, phenylacetylene (102 mg, $4 \mathrm{mmol}$ ) was quickly added under nitrogen atmosphere, and the mixture was heated to reflux for $30 \mathrm{~h}$. After the reaction was completed, the mixture was cooled to room temperature, and the crude product was concentrated. The residue was purified by silica gel column chromatography and recrystallized from chlorobenzene to give $300 \mathrm{mg}$ of DPEN (yield 45\%). MS (MALDI-TOF): 329.13. ${ }^{1} \mathrm{H}$ NMR (400 $\mathrm{MHz}, \mathrm{CDCl}_{3}$ ): $\delta 8.03(\mathrm{~s}, 2 \mathrm{H}), 7.79(\mathrm{~d}, 2 \mathrm{H}), 7.61-7.57(\mathrm{~m}, 6 \mathrm{H}), 7.41-7.36(\mathrm{~m}, 6 \mathrm{H})$. Anal. calcd for $\mathrm{C}_{26} \mathrm{H}_{16}$ : C, 95.09; H, 4.91. Found: C, 91.82; H, 4.83 .

\section{Conclusions}

In summary, a series of novel naphthalene derivatives (DIN, DSN and DPEN) were synthesized. These compounds show high stability, which was confirmed by TGA, UV-vis spectra and cyclic voltammetry. The morphology and crystallinity of the semiconductor thin films were characterized by AFM and XRD, respectively. A high mobility of up to $0.53 \mathrm{~cm}^{2} \mathrm{~V}^{-1} \mathrm{~s}^{-1}$ was achieved for the thin film transistor of DSN, which is higher by one to two orders of magnitude than that of the DIN and DPEN. Furthermore, two-component bilayer OFET devices were prepared by combining DSN with $\mathrm{F}_{16} \mathrm{CuPc}$ as the p-type and ntype material, respectively. Ambipolar performance was obtained by adjusting the device preparation procedure, with hole and electronic mobilities of up to $10^{-2} \mathrm{~cm}^{2} \mathrm{~V}^{-1} \mathrm{~s}^{-1}$. Furthermore, the electrical performance was increased to $0.73 \mathrm{~cm}^{2} \mathrm{~V}^{-1}$ $\mathrm{s}^{-1}$ using a two-component bilayer configuration. These results suggest that the two-component bilayer configuration not only can achieve ambipolar OFETs but also could contribute to enhancing the electrical performance compared to singlecomponent devices.

\section{Conflicts of interest}

There are no conflicts to declare.

\section{Acknowledgements}

The authors acknowledge financial support from the National Natural Science Foundation of China (21272049), the Ministry of Science and Technology of China (2013CB933500), and the Project for the Innovation of High Level Returned Overseas Scholars (or team) in Hangzhou, Beijing Science and Technology Project (code Z201100008420019).

\section{Notes and references}

1 H. Yan, Z. Chen, Y. Zheng, C. Newman, J. R. Quinn, F. Dotz, M. Kastler and A. Facchetti, Nature, 2009, 457, 679-686.

2 W. Hu, Organic Optoelectronics, Wiley-VCH, 2013.
3 W. Wu, Y. Liu and D. Zhu, Chem. Soc. Rev., 2010, 39, 14891502.

4 C. Wang, H. Dong, W. Hu, Y. Liu and D. Zhu, Chem. Rev., 2012, 112, 2208-2267.

5 H. Dong, C. Wang and W. Hu, Chem. Commun., 2010, 46, 5211-5222.

6 K. Kobashi, R. Hayakawa, T. Chikyow and Y. Wakayama, Nano Lett., 2018, 18, 4355-4359.

7 R. P. Ortiz, A. Facchetti and T. J. Marks, Chem. Rev., 2010, 110, 205-239.

8 S. Guo, J. Yao, Y. Wang, L. Zhang, F. Zhai, X. Zhang, Y. Feng, W. Feng, X. Zhang, J. Jie, F. Yang, R. Li and W. Hu, J. Mater. Chem. C, 2021, 9, 5758-5764.

9 S. Z. Bisri, C. Piliego, J. Gao and M. A. Loi, Adv. Mater., 2014, 26, 1176-1199.

10 J. Zaumseil and H. Sirringhaus, Chem. Rev., 2007, 107, 12961323.

11 L. Wang, C. Wang, X. Yu, L. Zheng, X. Zhang and W. Hu, Sci. China Mater., 2020, 63, 122-127.

12 A. Dodabalapur, H. Katz, L. Torsi and R. Haddon, Science, 1995, 269, 1560-1562.

13 T. Taguchi, F. Chiarella, M. Barra, F. Chianese, Y. Kubozono and A. Cassinese, ACS Appl. Mater. Interfaces, 2021, 13, 86318642.

14 K. N. N. Unni, A. K. Pandey, S. Alem and J. M. Nunzi, Chem. Phys. Lett., 2006, 421, 554-557.

15 S. D. Wang, K. Kanai, Y. Ouchi and K. Seki, Org. Electron., 2006, 7, 457-464.

16 J. Shi, H. Wang, D. Song, H. Tian, Y. Geng and D. Yan, Adv. Funct. Mater., 2007, 17, 397-400.

17 J. Wang, H. Wang, X. Yan, H. Huang and D. Yan, Chem. Phys. Lett., 2005, 407, 87-90.

18 K. Eguchi, M. M. Matsushita and K. Awaga, J. Phys. Chem. C, 2018, 122, 26054-26060.

19 X. Zhou, Z. Wang, R. Song, Y. Zhang, L. Zhu, D. Xue, L. Huang and L. Chi, J. Mater. Chem. C, 2021, 9, 1584-1592.

20 H. S. Kim, Y. H. Kim, T. H. Kim, Y. Y. Noh, S. Pyo, M. H. Yi, D. Y. Kim and S. K. Kwom, Chem. Mater., 2007, 19, 35613567.

21 H. Tian, J. Shi, D. Yan, L. Wang, Y. Geng and F. Wang, Adv. Mater., 2006, 18, 2149-2152.

22 H. Tian, J. Wang, J. Shi, D. Yan, L. Wang, Y. Geng and F. Wang, J. Mater. Chem., 2005, 15, 3026-3033.

23 F. Juraj, M. Róbert, C. Marek, W. Martin, J. Ján, G. Katarína, P. Milan, D. Edmund and P. Martin, Synth. Met., 2015, 202, 73-81.

24 J. K. Myeong, M. Eigo, O. Itaru, T. Kazuo and N. Akiko, ACS Appl. Mater. Interfaces, 2013, 5, 2331-2336.

25 A. Minoru, Y. Koichi, F. Atsuko, K. Reizo, H. Kouji and T. Jun, Chem. Mater., 2008, 20, 4883-4890.

26 Q. Zhao, T. H. Kim, J. W. Park, S. O. Kim, S. O. Jung, J. W. Kim, T. Ahn, Y. Kim, M. Yi and S. Kwon, Adv. Mater., 2008, 20, 4868-4872.

27 A. Pron, P. Gawrys, M. Zagorska, D. Djuradoa and R. Demadrille, Chem. Soc. Rev., 2010, 39, 2577-2632.

28 L. Lu, P. Chellan, G. S. Smith, X. Zhang, H. Yan and J. Mao, Tetrahedron, 2014, 70, 5980-5985. 
29 S. Shinamura, I. Osaka, E. Miyazaki, A. Nakao, M. Yamagishi, J. Takeya and K. Takimiya, J. Am. Chem. Soc., 2011, 133, 5024-5035.

30 S. Shinamura, E. Miyazaki and K. Takimiya, J. Org. Chem., 2010, 75, 1228-1234.

31 T. Kamikawa and T. Hayashi, J. Org. Chem., 1998, 63, 89228925.
32 H. Meier, E. Praß, R. Zertani and H. L. Eckes, Chem. Ber., 1989, 122, 2139-2146.

33 A. E. Siegrist, P. Liechti, H. R. Meyer and K. Weber, Helv. Chim. Acta, 1969, 52, 253-254.

34 G. Dai, J. Chang, L. Jing and C. Chi, J. Mater. Chem. C, 2016, 4, 8758-8764.

35 C. Chi and G. Wegner, Macromol. Rapid Commun., 2005, 26, 1532-1537. 